\title{
RECONSTRUÇÃO FARINGOESOFÁGICA COM RETALHO LIVRE DE JEJUNO APÓS FARINGOLARINGOESOFAGECTOMIA CERVICAL
}

\author{
PHARYNGOESOPHAGEAL RECONSTRUCTION USING FREE JEJUNAL FLAP \\ FOLLOWING NECK PHARYNGOLARYNGOESOPHAGECTOMY
}

\author{
Mário Sergio Lomba Galvão, TCBC-RJ ${ }^{1}$ \\ Paulo Roberto Albuquerque Leal, TCBC-RJ' ${ }^{2}$ \\ Juliano Carlos Sbalchiero, ACBC-R $\mathbf{J}^{3}$ \\ Fernando Luiz Dias, TCBC-J ${ }^{4}$ \\ Terence Pires de Farias, ACBC-RJ ${ }^{5}$ \\ Max Jefferson Marques e Marques, RCBC-RJ ${ }^{6}$
}

\begin{abstract}
RESUMO: Objetivo: Os autores apresentam os resultados de reconstruções esofágicas com retalho microcirúrgico de jejuno obtidos no Instituto Nacional de Câncer entre dezembro de 1998 e março de 2001, ressaltando suas vantagens, desvantagens e suas complicações. Método: Foram estudados sete pacientes portadores de neoplasias avançadas de laringe, faringe ou da tireóide que foram submetidos no Instituto Nacional de Câncer (Rio de Janeiro-Brasil) à reconstrução esofágica cervical com interposição de retalho livre de jejuno após faringolaringectomia associada à esofagectomia cervical. Foram cinco homens e duas mulheres com uma média de idade de 54 anos (39-66). Três pacientes foram submetidos à reconstrução esofágica imediata e em quatro casos a reconstrução foi tardia, em média após 10,5 meses. Resultados: Não existiram perdas do retalho ou mortes no período pósoperatório avaliado. Das complicações, a mais freqüente foi infecção de ferida operatória $(57,1 \%)$. Fístulas salivares acometeram dois casos $(28,5 \%)$ e necessitaram de tratamento cirúrgico. Outras complicações incluíram estenose $(14,1 \%)$ e hipopafatireoidismo $(28,5 \%)$. Não houve complicações abdominais. Quatro dos sete casos apresentaram recidiva da doença em um período médio de 16,6 meses. Cinco pacientes reconquistaram uma satisfatória capacidade de deglutição após o procedimento. Conclusão: A experiência apresentada sugere que o retalho livre de jejuno consiste em um procedimento seguro, com elevados índices de sucesso para reabilitação da via digestiva e com baixos índices de complicações se comparado a outros métodos.
\end{abstract}

Descritores: Retalho livre de jejuno; Esofagectomia cervical; Faringoesôfago; Reconstrução faringoesofágica; Ressecção faringoesofágica.

\section{INTRODUÇÃO}

Pacientes portadores de carcinomas avançados da laringe, hipofaringe e esôfago no nível cervical, candidatos a tratamento cirúrgico, requerem um procedimento radical que envolve a criação de uma traqueostomia terminal definitiva e uma solução de continuidade entre a cavidade oral e o esôfago torácico quando não é possível a reconstituição primária da via digestiva.

O restabelecimento da continuidade do trato digestivo e, por conseguinte da via oral, apresenta um profundo impacto na qualidade de vida destes pacientes, evitando

1. Membro Titular da Sociedade Brasileira de Cirurgia Plástica

2. Membro Titular da Sociedade Brasileira de Cirurgia Plástica; Fellow do American College of Surgeons; Chefe do Serviço de Cirurgia Plástica Reparadora e Microcirurgia do INCa

3. Membro Titular da Sociedade Brasileira de Cirurgia Plástica; Médico do Serviço de Cirurgia Plástica Reparadora e Microcirurgia do INCa

4. Fellow do American College of Surgeons; Chefe do serviço de Cirurgia de Cabeça e Pescoço do INCa

5. Médico do Serviço de Cirurgia de Cabeça e Pescoço do INCa

6. Médico residente do Serviço de Cirurgia Plástica Reparadora e Microcirurgia do INCa

Recebido em 17/12/2001

Aceito para publicação em 09/07/2002

Trabalho realizado no Departamento de Cirurgia Plástica Reparadora e Microcirurgia e no Departamento de Cirurgia de Cabeça e Pescoço do Instituto Nacional de Câncer — INCa, Rio de Janeiro 
uma situação de fístula orocervical com sialorréia constante e a própria privação da gustação.

Ressaltando que estes pacientes têm uma sobrevida média de cinco anos de aproximadamente 25-35\% após a ressecção ${ }^{1}$, é imperativa a necessidade de um método que apresente pouca morbidade e que restaure a via oral de alimentação no menor tempo possível.

Entre as variadas técnicas para reconstrução do esôfago cervical, a que utiliza retalho livre de jejuno tem sido muito recomendada, com bons resultados apresentados por vários autores ${ }^{2-5}$.

Esta técnica foi inicialmente proposta por Seidenberg e cols. ${ }^{6}$ em 1959. Naquela época, as técnicas e materiais de cirurgia microvascular eram pouco desenvolvidos, o que impedia a conquista de bons resultados. Desde então foram utilizados procedimentos em múltiplos estágios para este fim, como retalhos pediculados fasciocutâneos (deltopeitoral), miocutâneos (peitoral maior) e posteriormente interposição de vísceras. Com o desenvolvimento da microcirurgia a partir de meados da década de 70, o retalho livre de jejuno se tornou um método acessível e efetivo para reconstrução da continuidade entre a faringe e o esôfago cervical. Vale salientar que o reconhecimento das vantagens dos retalhos microcirúrgicos para reconstrução faringoesofágica tem estimulado o desenvolvimento de outras alternativas, como o retalho livre antebraquial tubulizado ${ }^{6}$.

Neste artigo os autores apresentam os resultados do Serviço de Cirurgia Plástica Reparadora e Microcirurgia do Instituto Nacional de Câncer (Rio de Janeiro - Brasil) em sete casos de reconstruções esofágicas através de transplante de jejuno microcirúrgico, relatando ainda os benefícios e as complicações relacionadas à técnica.

\section{MÉTODO}

Foram analisados retrospectivamente seis pacientes portadores de carcinoma epidermóide de laringe com acometimento da hipofaringe e um paciente portador de adenocarcinoma papilífero da tireóide operados no período compreendido entre dezembro de 1998 e março de 2001. Todos apresentavam tumores localmente avançados e foram submetidos a laringectomia total com hipofaringectomia total e esofagectomia cervical associada a esvaziamento cervical bilateral (lateral ou radical).

A reconstrução com retalho microcirúrgico de jejuno foi realizada tardiamente em quatro casos, em média após 10,5 meses (Figura 1). Nesses pacientes, devido a achados intra-operatórios incidentais e impossibilidade de reconstrução imediata, foi realizada sutura em fundo cego da orofaringe além de esofagostomia cervical.

Os pacientes incluíam cinco homens e duas mulheres, com uma idade média de 54,1 anos (39-66). Cinco deles eram tabagistas $(71,4 \%)$ e quatro eram alcoólatras. Pacientes submetidos à reconstrução tardia sofreram radioterapia prévia que variou entre 45 a $65 \mathrm{~Gy}$, em média, seis meses antes do procedimento reconstrutor.

As cirurgias foram realizadas envolvendo uma equipe multidisciplinar composta por membros da cirurgia de cabeça e pescoço, cirurgia plástica e cirurgia abdominal.

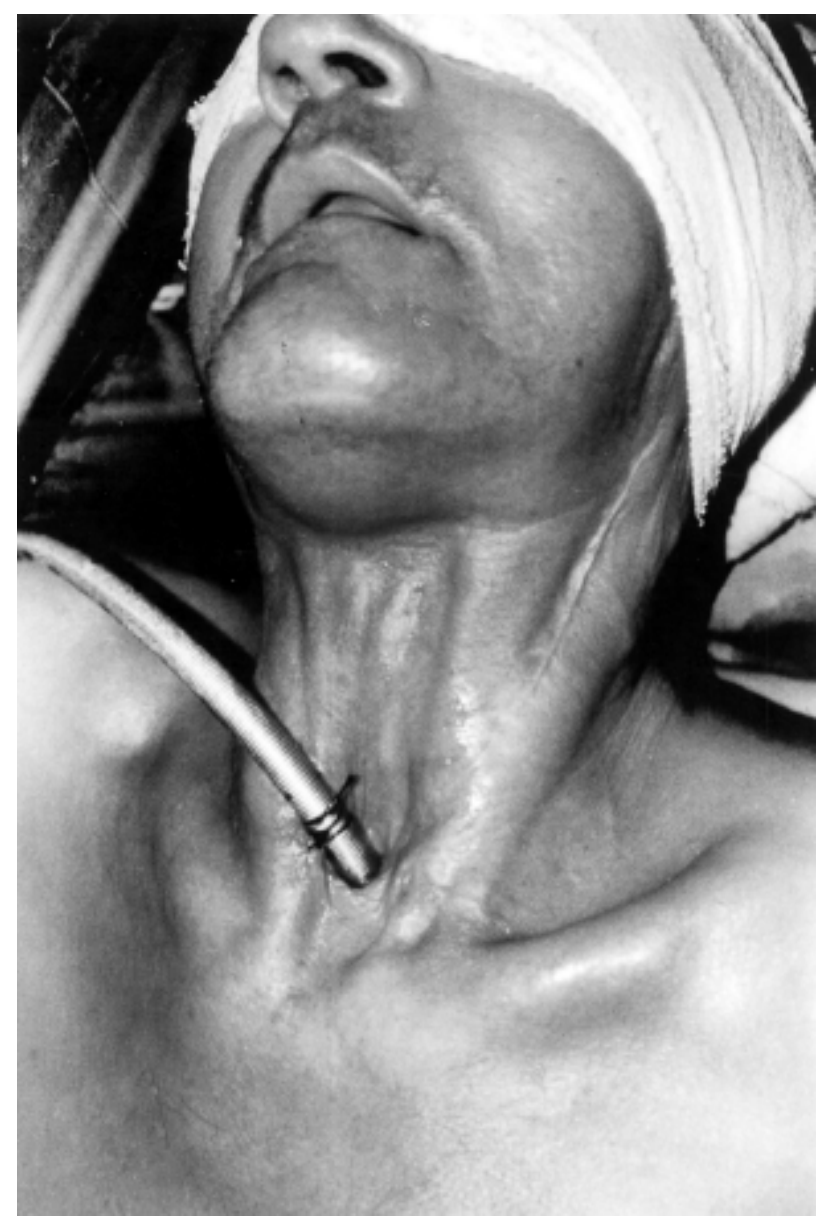

Figura 1 - Pré-operatório de reconstrução esofágica tardia após faringolaringoesofagectomia.

Os pacientes foram submetidos à profilaxia antibiótica com cefazolina e metronidazol durante 72 horas, iniciados durante indução anestésica. Punções das veias subclávia ou jugular interna foram contra-indicadas em decorrência do esvaziamento cervical e das anastomoses microcirúrgicas.

Após faringolaringectomia associada à esofagectomia cervical, a equipe de cirurgiões de Cabeça e Pescoço isola os cotos faríngeo e esofágico (Figura2). Simultaneamente o paciente é submetido a laparotomia, sendo isolado um segmento de cerca de $20 \mathrm{~cm}$ de alça jejunal a aproximadamente $30-40 \mathrm{~cm}$ do ângulo de Treitz, após verificação por transiluminação das arcadas vasculares e identificação do pedículo doador.

As extremidades proximal e distal deste segmento são então seccionadas com sutura mecânica, deixando-o perfundido pelo seu pedículo (Figura 3). A seguir, o segmento jejunal é transplantado para o pescoço após prévia ligadura dos vasos mesentéricos no abdômen, sendo o retalho posicionado em posição isoperistáltica. Com utilização de microscópio cirúrgico, realiza-se a anastomose vascular com fios de náilon 8-0 a 10-0, na dependência do calibre vascular. Em quatro pacientes o pedículo receptor 
consistiu na artéria carótida externa e veia jugular interna por meio de anastomose terminolateral. Três desses casos foram submetidos à reconstrução tardia. Nos outros casos, foram utilizados como pedículos receptores a artéria e veia cervical transversa, a artéria e veia tireoidiana superior e o tronco linguofacial por meio de anastomose terminoterminal.

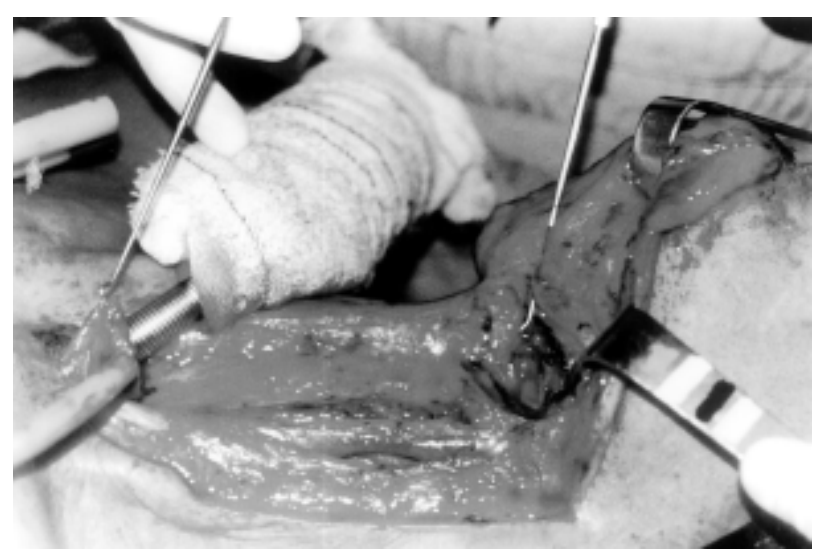

Figura 2 - Cotos faríngeo e esofágico após dissecção cirúrgica.

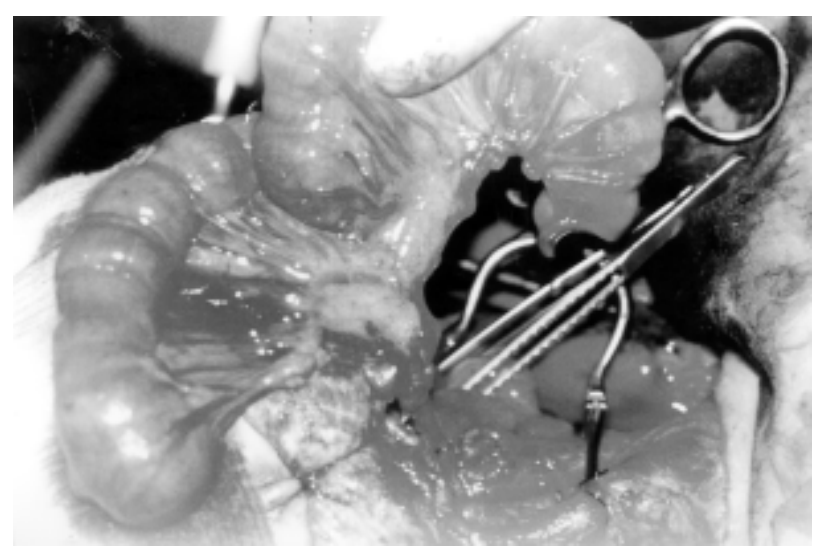

Figura 3 - Segmento jejunal seccionado, perfundido pelo seu pedículo.

O próximo passo consiste na anastomose faríngea, realizada com pontos separados, seromusculares de fio de poliglactina 3-0, em plano único, com inclusão posterior da fáscia pré-vertebral para maior suporte do retalho. Nesse instante, uma sonda nasoenteral é passada através do retalho. Devido à discrepância entre o lúmen jejunal e o defeito faríngeo, em cinco casos a anastomose foi terminolateral e nos outros dois terminoterminal. A anastomose jejunoesofágica foi sempre realizada de forma terminoterminal em plano único com pontos separados de Poliglactina 3-0, evitando-se redundância da alça jejunal (Figura 4). A enteroanastomose na área doadora é realizada em plano único de poliglactina 3-0 pela equipe da cirurgia abdominal.

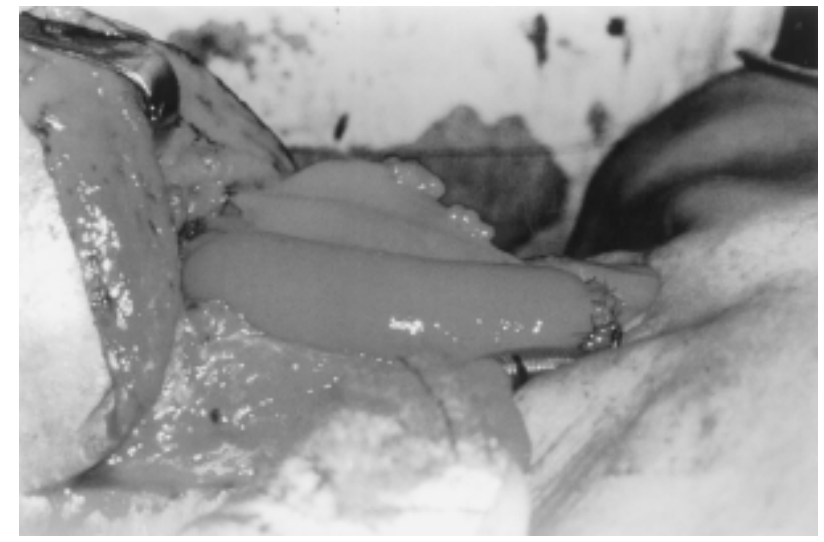

Figura 4 - Segmento jejunal após conclusão das anastomoses.

Realiza-se drenagem fechada do sítio cirúrgico, evitando a proximidade dos drenos com as anastomoses vasculares.

Em um dos casos de reconstrução tardia, submetido à radioterapia prévia, houve necessidade de confecção de retalho fasciocutâneo deltopeitoral à esquerda para cobertura cutânea em região cervical (Figura 5).

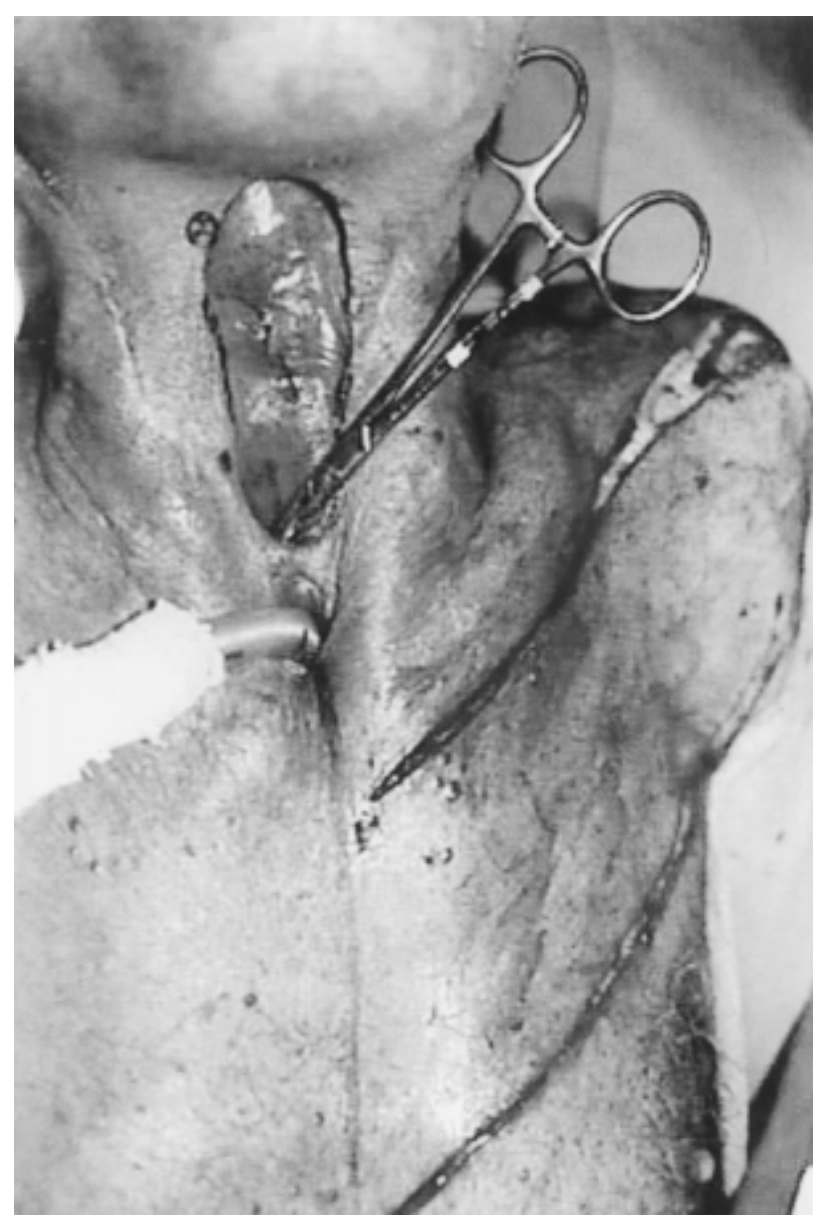

Figura 5 - Retalho fasciocutâneo deltopeitoral para reconstrução de defeito cutâneo em região cervical anterior. 
No manejo pós-operatório, esses pacientes foram observados durante o primeiro dia na unidade de terapia intensiva. Cuidados foram tomados no intuito de manter a cabeça do paciente em leve rotação para o lado contralateral à anastomose vascular com o objetivo de evitar qualquer dobra do pedículo. A cabeceira foi mantida elevada a $30^{\circ}$. Qualquer compressão extrínseca na região cervical foi evitada.

A monitorização da viabilidade do retalho foi realizada por visualização direta com laringoscopia de fibra óptica no $1^{\circ}$ dia pós-operatório (DPO). Em um caso foi utilizada uma "janela" na ferida cirúrgica com visualização direta da coloração da alça.

A dieta por sonda nasoenterai foi iniciada a partir do $2^{\circ}$ DPO, na dependência de peristalse intestinal propulsiva. A dieta oral foi iniciada de forma progressiva em torno do $10^{\circ}$ DPO, após realização de esofagograma com contraste não baritado e ausência de complicações fistulosas (Figura 6). Durante a internação, dosagens séricas do cálcio foram realizadas devido à possibilidade de hipoparatireoidismo.

Após alta hospitalar, esses pacientes foram acompanhados por uma equipe multidisciplinar composta por cirurgiões, clínicos endocrinologistas, nutricionistas e fo-

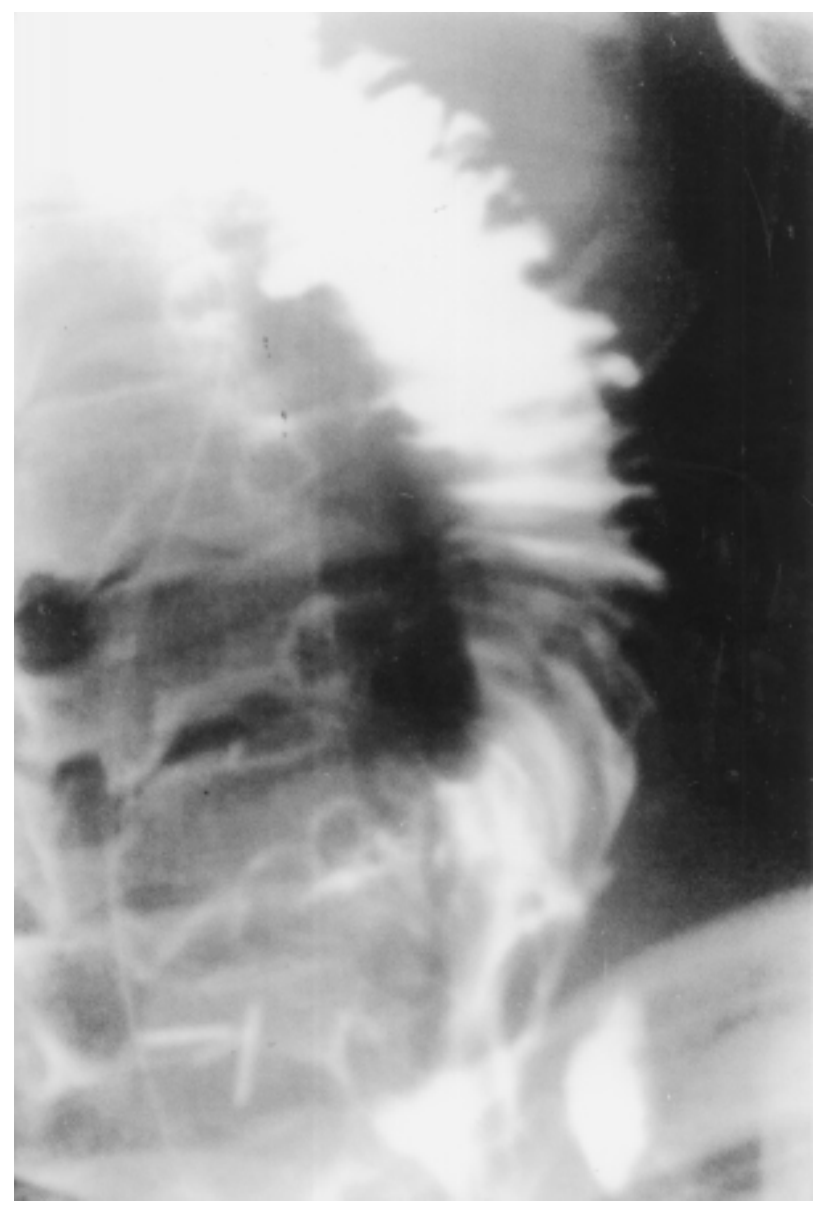

Figura 6 - Estudo contrastado do segmento jejunal transplantado, observando-se satisfatória progressão do contraste. noaudiólogos, responsáveis pela reabilitação da voz e da deglutição, uma vez que nesses pacientes o mecanismo da deglutição foi perdido e esta se faz apenas de forma passiva.

Todos os pacientes foram submetidos à radioterapia após a ressecção tumoral.

\section{RESULTADOS}

Sete pacientes foram submetidos à reconstrução faringoesofágica sem nenhuma perda do retalho, portanto com uma taxa de sucesso de $100 \%$. Não houve mortalidade perioperatória.

Com base na capacidade de manter o peso corpóreo sem necessidade de sondas para alimentação e na habilidade de deglutir alimentos sólidos, pastosos ou líquidos, os pacientes tiveram sua função de deglutição classificada em níveis ótimo, bom, regular e insatisfatório quando houve necessidade de sonda para alimentação. Três pacientes $(43 \%)$ apresentaram uma ótima função de deglutição. Dois deles $(28,5 \%)$ apresentaram uma função de deglutição considerada boa, com pequena disfagia para alimentos sólidos. Em um caso (14,2\%), a função foi regular e em outro houve necessidade de sonda para alimentação $(14,2 \%)$.

O inicio da dieta por via oral foi realizado em média no $11^{\circ}$ DPO (10-20을 nos casos em que não houve complicações pós-operatórias significativas.

A complicação mais freqüente foi a infecção de ferida operatória, observada em quatro casos $(57,1 \%)$. Dois desses pacientes evoluíram com fístula salivar $(28,1 \%)$ de alto débito, um dos casos irradiado previamente. Ambas estavam localizadas na anastomose distal, sendo necessário tratamento cirúrgico para sua correção.

Dois pacientes $(28,5 \%)$ evoluíram no pós-operatório com hipoparatireoidismo. Foram submetidos à reposição de cálcio e estiveram sob acompanhamento do serviço de endocrinologia. Quatro pacientes submetidos a tireoidectomia total $(57,1 \%)$ necessitaram de reposição de hormônio tireoidiano.

Apenas um paciente evoluiu com estenose tardia na altura da anastomose proximal, tendo sido submetido à dilatação endoscópica com razoável melhora do quadro. Este paciente não apresentava história de fístula associada e representava um caso de reconstrução imediata.

Não existiram complicações abdominais, torácicas ou sistêmicas nestes pacientes.

A permanência hospitalar pós-operatória foi em média de 54 dias (22 -86) para os pacientes que apresentaram fístula salivar (dois casos) e de 12 dias (10 -14) para o restante.

Em relação ao controle oncológico, três pacientes sobrevivem sem evidência de recidiva tumoral com 17, 12 e 57 meses de acompanhamento. O primeiro caso corresponde a uma paciente portadora de adenocarcinoma papílifero da tireóide. Os outros dois foram casos de carcinoma epidermóide de laringe sem linfonodos metastáticos. Nos outros pacientes, a recidiva da doença aconteceu em média após 16,6 meses da ressecção tumoral. 


\section{DISCUSSÃO}

A eficiência deste procedimento de reconstrução está na oportunidade dada ao paciente de recuperar parcialmente a função de deglutição perdida em um período de tempo satisfatório em relação à história natural de sua doença, com níveis de complicações aceitáveis.

O sucesso de $100 \%$ na viabilidade do jejuno transplantado, assim como em outras séries ${ }^{347}(85-100 \%)$, traduz a aplicabilidade do método para reconstrução esofágica cervical. Além da segurança, outros fatores nos incentivam a utilizá-lo: facilidade de exposição, vascularização abundante, resistência da sua parede, boa lubrificação e flora bacteriana relativamente benigna associada à possibilidade de repetição do procedimento devido ao sítio doador abundante ${ }^{8}$.

Além disso, sabemos que esses pacientes têm uma sobrevida curta, em média, menor que dois anos ${ }^{1}$, mesmo quando realizadas ressecções radicais com intenções curativas. A recidiva da doença está relacionada principalmente com o envolvimento linfonodal regional ${ }^{9}$. Como foi visto na nossa série, recidivas da doença ocorreram em média após 16,6 meses da ressecção e por isso ressaltamos a necessidade de um procedimento que seja realizado em um único tempo cirúrgico ${ }^{10}$, com reabilitação da sua função em um curto período de tempo. Os casos avaliados que não apresentaram complicações iniciaram dieta oral em média no 110 DPO e tiveram altas hospitalares por volta do 120 DPO, condizente com os dados de outros autores ${ }^{1,3,7}$. Em $71 \%$ dos casos as queixas de disfagia não foram significativas, também equivalentes a dados da literatura ${ }^{1,2}$.

Comparando com a transposição gástrica ou colônica, nestes métodos o tórax é violado e a morbidade resultante das complicações pulmonares junto aos riscos de infecção mediastinal e pleural são muito maiores ${ }^{5}$, além da limitação de alcance do pedículo vascular.

Retalhos pediculados fasciocutâneos ou miocutâneos como o deltopeitoral e o peitoral maior não são tão seguros quanto o transplante jejunal e apresentam a des- vantagem da maior rigidez para confecção de um retalho tubular, necessidade de múltiplos estágios cirúrgicos, ausência de autolubrificação associada à descamação cutânea, além da maior morbidade para a área doadora ${ }^{3}$. Nesses retalhos a incidência de fístulas varia de $40 \%$ a $58 \%{ }^{3,6}$. São normalmente fístulas proximais de difícil manejo com tratamento conservador ${ }^{5,6}$. Em relação ao retalho livre antebraquial, apesar da relativa segurança, apresenta um índice de fístulas elevado, provavelmente decorrente da extensa linha de sutura $^{6}$ necessária para modelagem da forma tubular.

A incidência de fístula neste estudo foi de $28,5 \%$, taxa semelhante à de outras séries ${ }^{1,5,7}$. Porém, ao contrário do que aconteceu com nossos pacientes, as fístulas são normalmente proxímais ${ }^{2,9,11}$ e apesar da necessidade de tratamento cirúrgico, a maioria delas é resolvida com condutas conservadoras ${ }^{1,11}$.

Vale salientar que as complicações foram mais freqüentes nos pacientes submetidos à radioterapia prévia. Nestes casos, tanto o acesso cirúrgico quanto a anastomose vascular microcirúrgica apresentaram maior dificuldade técnica. Em função disto, indicamos sempre que possível a reconstrução imediata. Dois dos três pacientes reconstruídos imediatamente não tiveram qualquer complicação.

Dois pacientes do estudo apresentaram queixas de disfagia significativa, justificada em um dos casos por estenose no nível da anastomose proximal. O outro paciente não apresentou estenose ou sinais de redundância jejunal, podendo ser atribuída a causas nãoanatômicas ${ }^{5}$. É importante salientar que algum grau de edema permanece por alguns meses e este pode resultar em queixas de disfagia leve relatada por alguns pacientes ${ }^{2}$.

Enfim, a nossa experiência com os casos operados sugeriu que a reabilitação precoce associada aos índices relativamente baixos de complicações confirmam a efetividade do retalho livre de jejuno que permanece como procedimento de primeira escolha em nosso serviço para reconstrução esofágica cervical.

\begin{abstract}
Objective: The present study describes the results at the Instituto Nacional de Câncer (Rio de Janeiro-BRAZIL) in reconstruction of cervical esophagus using jejunal interpositional flap. Advantages, disadvantages and possible complications are discussed. Method: Seven patients with Iocally advanced carcinoma of the Iarynx and thyroid underwent pharyngoesophageal reconstruction using free jejunal interpositional flap after pharyngolaryngectomy-esophagectomy from december/1 998 through march/2001 at Instituto Nacional de Cancer. There were five men and two women with mean age of 54,1 years old (39-66 years). Three patients underwent immediate esophageal reconstruction. In four cases, the reconstructions were performed under delayed basis (in mean, after 10,5 months). Results: There were no flap failures or perioperative deaths. The most frequent complication was wound infection (57,1\%). Salivary fistula occured in two cases $(28,5 \%)$ and required secundary revision. Other complications were stenosis $(14,1 \%)$ and hypoparathyroidism $(28,5 \%)$. There were no abdominal complications. Four out of seven patients presented recurrent disease in a mean time of 8,5 months (3-13 months). Five patients (71\%) acquired a satisfactory swallow function after surgery. Conclusion: The cases here presented, suggest that free jejunal flap is a safe procedure with high success rates for high digestive tract reabilitation, with few complications.
\end{abstract}

Key Words: Jejunal free flap; Cervical esophagectomy; Pharyngoesophagus; Pharyngoesophageal reconstruction; Pharyngoesphageal resection. 


\section{REFERÊNCIAS}

1. Reece GP, Bengtson BP, Schusterman MA. Reconstruction of the pharynx and cervical esophagus using free jejunal transfer. Clin Plast Surg 1994; 21: 125-36.

2. Gluckman JL, McDonough JJ, McCafferty GJ et al. Complications associated with free jejunal graft reconstruction of the pharyngoesophagus - a multiinstitutional experience with 52 cases. Head Neck Surg 1985; 7: $200-5$.

3. Giavanoli P, Manfred F, Schmid S. Free jejunum transfers for functional reconstruction after tumors resections in the oral cavity and the pharinx: changes of morfology and function. Microsurgery 1996 17: 535-44.

4. Inoue Y, Tai Y, Fujita M et al. A retrospective study of 66 esophageal reconstructions using microvascular anastomoses: problems and our methods for atypical cases. Plast Reconstr Surg 1994; 94: 277-84.

5. Coleman JJ III, Searles JM, Hester TR et al. Ten years experience with free jejunal autograft. Am J Surg 1987; 154: 394-98.

6. Seidenberg B, Rosenak 88, Hurwitt ES et al. immediate reconstruction ofthe cervical esophagus by a revascularized isolated jejunal segment. Ann Surg 1959; 149: $162-171$

7. Coieman JJ III. Reconstruction of the pharynx and cervical esophagus. Seminars in Surgical Oncology 1995; 11:208-20.

8. Fisher RS, Cameron R. Free jejunal interpositon graft for reconstruction of the esophagus. Head Neck Surg 1990; march/April: 126-30.

9. Ferguson JL, De Sonto LW. Total pharyngolaringectomy and cervical esophagectomy with jejunal autotransplant reconstruction: complications and results. Laringoscope 1988; 98: 911-14
10. Omura K, Misaki T, Watanabe $\mathrm{Y}$ et al. Reconstruction with free jejunal autograft after pharyngolaryngoesophagectomy. Ann Thorac Surg 1994; 57:112-17.

11. Sasaki TM, Baker HW, McConneii DB et al. Free jejunal graft reconstruction after extensive head and neck surgery. Am J. Surg 1980;139: 650-53.

12. Galvão MSL, Junior HBL. Reconstrução da faringe e do esôfago cervical. In Dias FL e Noronha M Jr. Câncer da laringe: uma abordagem muitidisciplinar. Primeira edição. Rio de Janeiro. Editora Revinter, 2001, pp.204214.

Endereço para correspondência:

Max Jefferson Marques e Marques

Rua Washington Luis, 85/apto. 501

20230-021 - Rio de Janeiro-RJ

E-mail:maxmq@bol.com.br 